\title{
Dynamic modelling and parameter identification for cable-driven manipulator
}

\author{
Fei Yan $^{1}$, Yaoyao Wang ${ }^{1, *}$, Feng $\mathrm{Ju}^{1,2}$, Jiafeng $\mathrm{Yao}^{1}$, Bai Chen ${ }^{1}$ and Hongtao $\mathrm{Wu}^{1}$ \\ ${ }^{1}$ College of Mechanical and Electrical Engineering, Nanjing University of Aeronautics and Astronautics, Nanjing 210016, China \\ ${ }^{2}$ State Key Laboratory of Fluid Power and Mechatronic Systems, Zhejiang University, Hangzhou 310027, China
}

Cable-driven manipulators (CDMs) have several advantages, but unknown dynamic characters restrict their control performance. The motors of CDMs are placed at the base and power is transmitted by cables between the motors and driving pulleys. These cables interact with the links of the manipulator, which poses a challenge for obtaining the dynamic model. In this study, the interaction between cables and manipulators is analysed, and a dynamic model is derived based on Newton-Euler method. To eliminate excessive variance in recursive equations, the impact of pretension force is considered and some equivalent assumptions are proposed. To improve the accuracy of parameter identification, limited terms of Fourier series are adopted for the identification trajectory. Considering various limitations of CDMs, such as maximum joint angle, speed and acceleration, artificial bee colony algorithm is used to optimize the coefficients of the identification trajectory. Simulations verify that the dynamic model can precisely calculate the driving torque of CDMs. Moreover, parameter identification experiment affirms the efficiency of the proposed parameter identification method.

Keywords: Artificial bee colony algorithm, cable-driven manipulator, dynamics, parameter identification.

NOWADAYS, manipulators are widely used in mass production, medical purposes, and even in our daily lives. Traditional manipulators have their motors at the joints, which greatly increases their moving mass and inertia. Consequently, they are often isolated from humans for safety. Moreover, manipulators may suffer from malfunction due to a variety of reasons, such as program bug, mechanical abrasion and incorrect operation. Since they are increasingly vital in all walks of life, it is necessary for manipulators to share working space with humans. Thus, manipulators are expected to be light-weight considering human safety.

Cable-driven technique is an effective tool to decrease the moving mass and rotational inertia of manipulators ${ }^{1-4}$. By placing motors at the base and transmitting power through cables, a large amount of mass can be removed from the moving parts of manipulators ${ }^{5-7}$. Moreover,

*For correspondence. (e-mail: yywang_cmee@nuaa.edu.cn) the utility of cable-drive technique makes room for other devices, so cable-drive technique is extensively used in many fields such as exoskeleton.

There are a variety of studies dedicated to a wide range of cable-driven manipulators (CDMs). When cables are utilized to transfer driving force, there are nonlinear friction and nonlinear motion between links of manipulator and driving cables. Consequently, that phenomenon greatly impaires the motion accuracy of CDMs, especially when the driving cable is long-suspended. To tackle this problem, Du et al. ${ }^{8}$ performed the Jacobian analysis of a longspan cable-driven manipulator. Their method could deduce the Jacobian matrix that maps the infinitesimal change of the cable length coordinate to that of the endeffector coordinate, and could successfully find the forward solution with high precision ${ }^{8}$. Duan et al. ${ }^{9}$ studied the calibration and motion control of a triple-level spatial positioner consisting of a $50 \mathrm{~m}$ dimensional cable-driven parallel manipulator. After kinematic calibrations, the manipulator-based triple-level spatial positioner could achieve $2.55 \mathrm{~mm}$ RMS positioning and $0.06^{\circ}$ orientation accuracy ${ }^{9}$. Tzemanaki et al. ${ }^{10}$ designed a cable-driven mechanism as an affiliate instrument for an anthropomorphic master-slave system called $\mu$ Angelo. Their design followed the utilities of the human hand, such as supination, adduction and rotation. They proposed two ways of routing the cable-driven mechanism and found a method to derive the input-output functions of that mechanism. Gonzalez-Rodriguez et al. $^{11}$ proposed a concept to improve the connection between the fixed frame and endeffector of planar and spatial cable-driven robots. Their concept added pulleys to the attachment between the cables and the end-effector, and also included the reflective pulleys in the end-effector. Xue et al. ${ }^{12}$ proposed a tension-displacement transmission model for cablepulley system to tackle nonlinear friction and backlash characteristics of cable-driven mechanism in laparoscope surgical robots. They took the bending rigidity of the cable and spatial location of the pulleys into consideration, and designed a feed-forward controller using position compensation algorithm based on the model.

Establishing models of cable is another way to tackle their nonlinearity. Gungor et al. ${ }^{13}$ explored on-line estimation and compensation of friction in cable-driven parallel robots. They presented a recursive least-squares 
on-line friction estimator and an adaptive controller based on Lyapunov method. Francesco Fichera and co-workers proposed polynomial stiffness and polynomial elastic torque model for cable-based motor-to-joint transmissions ${ }^{14,15}$. They presented a more general elastic torque model based on polynomial stiffness, which can be applied to flexible-joint robots. Yu et al. ${ }^{16}$ proposed a new type of wire-driven device for surgical robots and designed an experiment model of a single joint to test the feasibility of the device. They considered the elasticity of the driven cable and proposed a dynamical model of the cable-driven finger joint. They also designed an estimator of the joint angle, and modelled the DC servo motor. Qi et $a l .{ }^{17}$ proposed an efficient model for the flexible cable with time-varying lengths and coupling motions between the cable and pulleys. Their model is useful in dynamic analysis of the cable-pulley system, but may be too complex for CDMs, which use a large number of pulleys. Proper trajectory is also significant for CDMs, considering the special nature of cables. Zhang and Shang ${ }^{18}$ proposed a geometrical approach to plan trajectories for a spatial 3-d of under-constrained cable-driven parallel robot. Their approach extended beyond the static equilibrium workspace of the mechanism, and could guarantee positive and continuous tension in the cables. Ismail et $a l .{ }^{19}$ proposed a dynamic path planning algorithm for hybrid cable-serial manipulator to find the shortest and fastest path which ensures bounded tensions in the actuator cables. They gave the trajectory through a geometric planning method, which ensures the shortest path between two poses of the robot while avoiding obstacles. Many other trajectory planning methods are also valuable to $\mathrm{CDMs}^{20-25}$.

Cables result in complicated dynamic character, which challenges the control performance of CDMs. Cabledrive is such a promising technique that many researchers are working on it. Lau et al. ${ }^{26}$ considered joint interaction forces and moments in the objective functions and constraints specific to the inverse dynamics of multilink CDMs. They formulated the relationship between the joint interactions and cable forces which included the constraints to maintain stability of unilateral spherical joints. Xuechao et al. ${ }^{27}$ proposed a dynamic model for cable-driven parallel manipulators with cables of slow time-varying lengths. They deduced the partial differential equation which characterized the dynamics of a cable with varying length. They also converted the partial differential equation into ordinary differential equations through spatial discretization by finite difference approximation. Nielsen et al. ${ }^{28}$ provided a mathematical description which captured most of the significant dynamics of underwater robots.

Controller design is another important issue of $\mathrm{CDMs}^{29-31}$. Several studies have been made to achieve satisfactory control performance for systems with complicated uncertainties $^{32-38}$. Kong and coworkers ${ }^{38,39}$ pro- posed a method of proxy-based impedance control as a rehabilitation algorithm of a cable-driven assistive system. They assumed free space around the proxy and free motion of a human joint was allowed in a certain range. Their methods applied assistive torque to compensate for the inertia mismatch and considered actuator dynamics for better implementation of rehabilitation algorithms. Since kinematic and dynamic models of cable-driven parallel robots are partly structurally unknown, Babaghasabha et $a l .^{40}$ proposed an adaptive, robust, sliding-mode controller based on the adaptation of the upper bound of the uncertainties. Caverly et $a{ }^{41}{ }^{41}$ designed a state estimation for single degree of freedom flexible cable-actuated system, and used a proportional derivative-based controller for control. They also estimated the tension in each cable, and considered sensors with different sampling rates. Jung et al. ${ }^{42}$ designed a cable-driven exoskeleton mechanism and addressed the problem of force control. They adopted a spring-actuator-type cable-driven mechanism to make the cable routing structure compact. They designed the controller by combining PD control algorithm which is optimized by a linear quadratic method with a disturbance observer and feed-forward filter to track zero-phase error. Boehler et al. ${ }^{43}$ proposed two control strategies for cable-driven tensegrity mechanism which allows one to control the angular position and the associated stiffness. The first strategy uses tension distribution algorithm which provides a modulation of stiffness, while the second strategy introduces a novel velocity distribution algorithm that allows for a modulation of stiffness derivative. Moreover, time-delay estimation technique and sliding mode control scheme are efficient tools in the controller design of $\mathrm{CDMs}^{44-48}$.

The model of CDM by Jin et al. ${ }^{49}$ only considers joint stiffness and damping force. Multilink-serial CDMs have cables passing through their joints; so it is inevitable that cables interact with manipulator mechanical links. In this study, interaction between cable and mechanical structure of the manipulator is considered and the dynamic model of cable-driven serial manipulator is derived based on the Newton-Euler method. Moreover, a parameter identification method is proposed and proper identification trajectory is devised. The dynamic model and parameter identification method are verified using MATLAB and ADAMS software. (1) Thus, the dynamic model of cabledriven serial manipulator is deduced considering the interaction between cables and mechanical structure of the manipulator. (2) Parameter identification method for $\mathrm{CDM}$ is proposed. Artificial bee colony algorithm is utilized to obtain the preferred identification trajectory.

\section{Basic structure and motion relationship}

Figure 1 shows the basic structure of the cable-driven serial manipulator and Figure 2 provides details of cable 
routing and guide pulley. In Figure 2, link guide pulley is fixed to the corresponding link. The centre of joint guide pulleys and joint drive pulleys correspond to the centre of the hinges between two joints.

First, we deduce some geometric relationships. Denote the radius of the joint guide pulley between link $i-1$ and link $i$ by $R$. One can easily see in Figure 1 that cables passing through a joint are under length variation when the angles between the links change. Specifically, if link $i$ rotates relative to link $i-1$ by angle $\theta$ in an anticlockwise direction, the cable on one side will wind onto the joint guide pulley while the cable on the other side will fall from the joint guide pulley, which appears as if the cable changes its length along with the rotation of links. Consequently, link $i+1$ will rotate relative to link $i$ in a clockwise direction. Given that the joint guide pulleys and joint drive pulleys have the same diameter, link

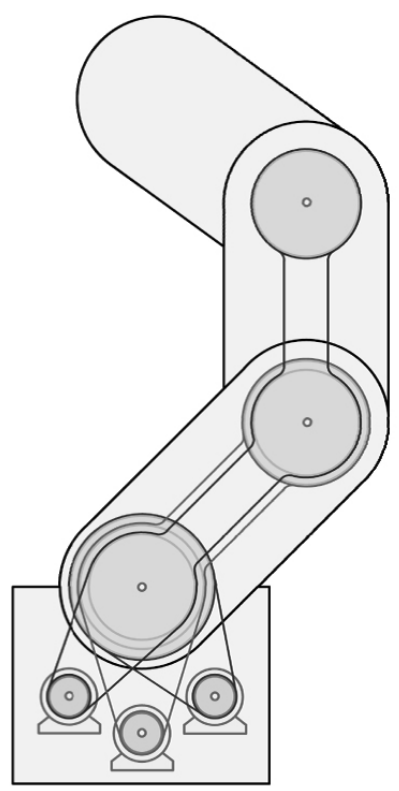

Figure 1. Basic structure of the cable-driven serial manipulator.

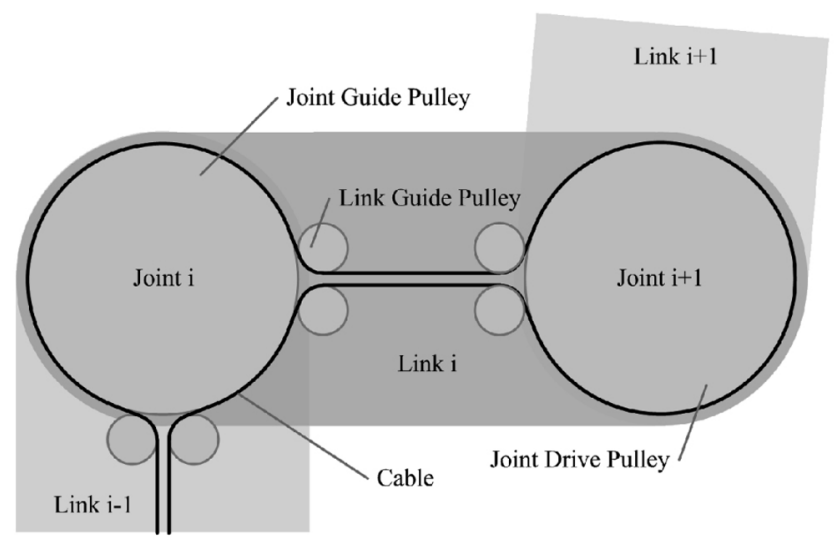

Figure 2. Details of cable routing and guide pulley. $i+1$ will rotate by angle $\theta$, the same angle that link $i$ rotates relative to link $i-1$. Under this condition, link $i+1$ translates without rotating relative to link $i-1$. Notice that all the joint guide pulleys and joint drive pulleys are of equal size, although they are of different size in Figure 1 for clear illustration.

Figure 3 shows the extreme position of joints in CDM. The radius of link guide pulley is denoted by $r_{\mathrm{g}}$; the radius of joint guide pulley and joint drive pulley are both $r_{j}$. The distance between the centre of link guide pulleys and centre of the links is $d_{\mathrm{g} 0}$. The line between joint guide pulley and link guide pulley is projected to coordinate axis $x_{i}$, and its length is denoted by $d_{j 0}$. For the extreme condition shown in Figure 3, cable 1 is tangent to the joint guide pulley. At this time, the angle between two links is $2 \theta_{\mathrm{j} 0}$. The extreme condition indicates that cable 1 will fall from the joint guide pulley if link $i$ rotates clockwise any further relative to link $i-1$. When cable 1 falls from the joint guide pulley, motion relationship would be changed and the robot would malfunction. One can get $\theta_{\mathrm{j} 0}$ from eq. (1) as follows

$$
\left(\frac{r_{\mathrm{g}} \cdot \sin \theta_{\mathrm{j} 0}-d_{\mathrm{g} 0}}{\cos \theta_{\mathrm{j} 0}}\right)^{2}+r_{\mathrm{g}}{ }^{2}=d_{\mathrm{g} 0}^{2}+\left(d_{\mathrm{j} 0}-\frac{r_{\mathrm{j}}}{\cos \theta_{\mathrm{j} 0}}\right)^{2} .
$$

It may be difficult to provide an analytical solution for $\theta_{\mathrm{j} 0}$, but a numerical solution is always available if other parameters are given. $\theta_{\mathrm{g} 0}$ is given by

$$
\theta_{\mathrm{g} 0}=\frac{\pi}{2}-\theta_{\mathrm{j} 0}
$$

Then the coordinate system will be established based on D-H method ${ }^{50}$. Figure 4 shows the coordinates for link $i$ and link $i+1$ without the $z$-axis, which can be determined by right-hand rule. In figure, the original coordinate system for link $i$ is located at the centre of joint $i$, where the centre of the hinge between link $i-1$ and link $i$ is located. The coordinate system $O_{i} x_{i} y_{i} z_{i}$, abbreviated as $O_{i}$, is fixed to link $i$. Link 0 denotes the base of the manipulator which is supposed to be fixed to the ground or a vehicle in uniform linear motion.

Let ${ }^{i} \omega_{i}$ denote the rotation vector of link $i$ relative to the coordinate system $i$, and similarly ${ }^{i+1} \omega_{i+1}$ for the rotation vector of link $i+1$ relative to coordinate system $i+1$. ${ }_{i}^{i+1} R$ is the transformation matrix from coordinate system $O_{i}$ to $O_{i+1}$.

$$
\begin{aligned}
& { }_{i}^{i+1} R=\left[{ }^{i+1} \hat{x}_{i} \quad{ }^{i+1} \hat{y}_{i} \quad{ }^{i+1} \hat{z}_{i}\right] \\
& =\left[\begin{array}{lll}
\hat{x}_{i} \cdot \hat{x}_{i+1} & \hat{y}_{i} \cdot \hat{x}_{i+1} & \hat{z}_{i} \cdot \hat{x}_{i+1} \\
\hat{x}_{i} \cdot \hat{y}_{i+1} & \hat{y}_{i} \cdot \hat{y}_{i+1} & \hat{z}_{i} \cdot \hat{y}_{i+1} \\
\hat{x}_{i} \cdot \hat{z}_{i+1} & \hat{y}_{i} \cdot \hat{z}_{i+1} & \hat{z}_{i} \cdot \hat{z}_{i+1}
\end{array}\right],
\end{aligned}
$$




\section{RESEARCH ARTICLES}

where $\hat{x}_{i}, \hat{y}_{i}$ and $\hat{z}_{i}$ are unit vectors relative to $x, y$ and $z$ axis respectively. ${ }_{i+i}^{i} R$ is given by

$$
{ }_{i+i}^{i} R={ }_{i}^{i+1} R^{-1}={ }_{i}^{i+1} R^{T}
$$

The pre-superscript $i+1$ indicates the reference coordinate system $O_{i+1} x_{i+1} y_{i+1} z_{i+1}$. $\theta_{i}$ is used to describe the angle between link $i-1$ and link $i$. Specifically, $\theta_{i}$ is defined as

$$
\left.\theta_{i}=\arcsin \left(\hat{x}_{i-1} \times \hat{x}_{i}\right) \cdot \hat{z}_{i}\right) .
$$

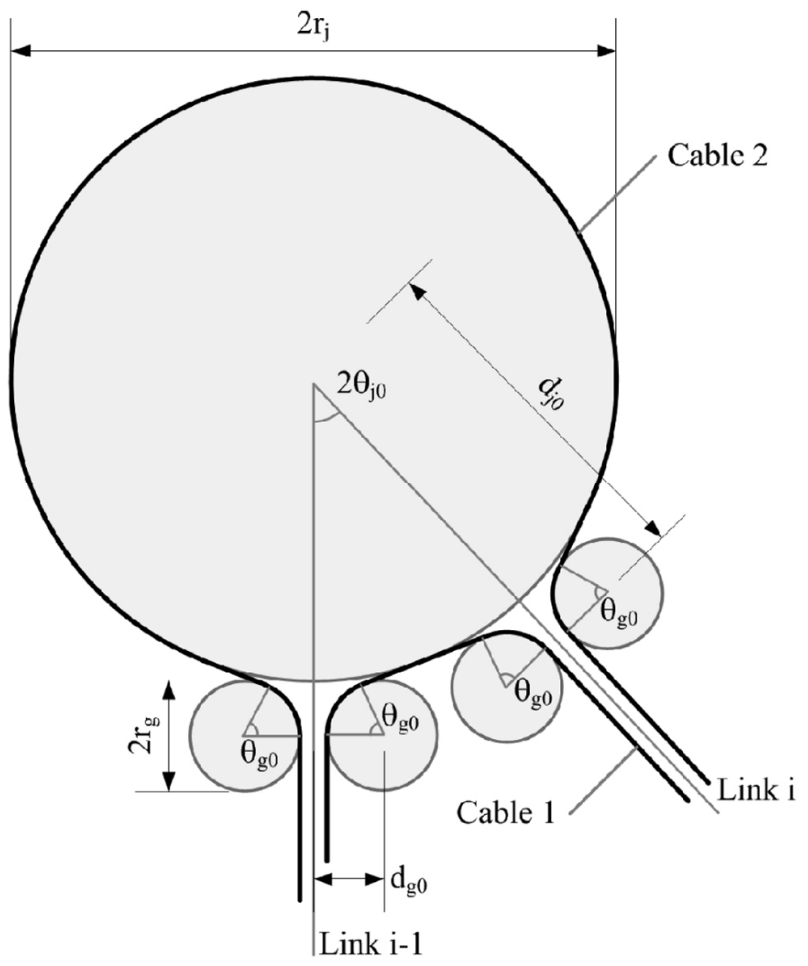

Figure 3. Extreme position of joints in CDM.

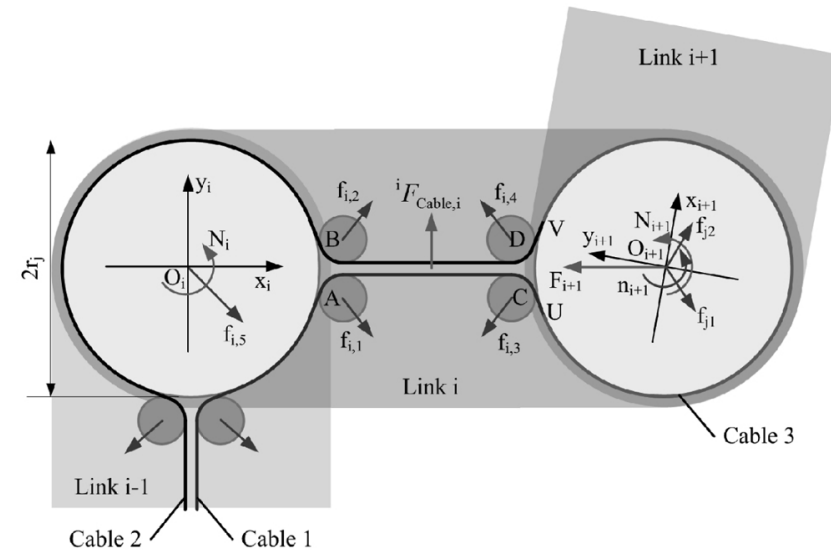

Figure 4. Coordinate for link $i$ and link $i+1$.
Consider that the orientation of a specific link is decided by its actuator in the cable-driven robot $^{51}$. Suppose the radius of the winches of motors is $r_{m} ; q_{i}, \dot{q}_{i}$ and $\ddot{q}_{i}$ represent the rotation angle, speed and acceleration of the motor of link $i$ respectively. $\theta_{i}$ is given by

$$
\theta_{i}=\frac{r_{\mathrm{m}}}{r_{\mathrm{j}}}\left(q_{i}-q_{i-1}\right)
$$

\section{Dynamic model}

Let ${ }^{i} P_{i+1}=\overrightarrow{O_{i} O_{i+1}}$ and ${ }^{i} P_{C, i}=\overrightarrow{O_{i} M_{i}}$, where $M_{i}$ is the mass centre of link $i$. The pre-superscript $i$ indicates that these vectors are measured in coordinate system $O_{i} \cdot{ }^{C, i+1} I_{i+1}$ is the inertia tensor matrix of link $i+1$ relative to its mass centre and ${ }^{i+1} \dot{v}_{C, i+1}$ is the velocity of the mass centre of link $i+1$. Kinematic recursion from $i$ to $i+1$ is given by the following equations:

$$
\begin{aligned}
{ }^{i+1} \omega_{i+1}= & { }_{i}^{i+1} R{ }^{i} \omega_{i}+\dot{\theta}_{i}{ }^{i+1} \hat{z}_{i+1}, \\
{ }^{i+1} \dot{\omega}_{i+1}= & { }_{i}^{i+1} R{ }^{i} \dot{\omega}_{i}+{ }^{i+1} R{ }^{i} \omega_{i} \times \dot{\theta}_{i+1}{ }^{i+1} \hat{z}_{i+1}+\ddot{\theta}_{i}{ }^{i+1} \hat{z}_{i+1}, \\
{ }^{i+1} \dot{v}_{i+1}= & { }_{i}^{i+1} R\left({ }^{i} \dot{\omega}_{i} \times{ }^{i} P_{i+1}+{ }^{i} \omega_{i} \times\left({ }^{i} \omega_{i} \times{ }^{i} P_{i+1}\right)+{ }^{i} \dot{v}_{i}\right), \\
{ }^{i+1} \dot{v}_{C, i+1}= & { }^{i+1} \dot{\omega}_{i+1} \times{ }^{i+1} P_{C, i+1}+{ }^{i+1} \omega_{i+1} \\
& \times\left({ }^{i+1} \omega_{i+1} \times{ }^{i+1} P_{C, i+1}\right)+{ }^{i+1} \dot{v}_{i+1}, \\
{ }^{i+1} F_{i+1}= & m_{i+1}{ }^{i+1} \dot{v}_{C, i+1}, \\
{ }^{i+1} N_{i+1}= & { }^{C, i+1} I_{i+1}{ }^{i+1} \dot{\omega}_{i+1}+{ }^{i+1} \omega_{i+1} \times{ }^{C, i+1} I_{i+1}{ }^{i+1} \omega_{i+1},
\end{aligned}
$$

where $r_{\mathrm{m}}$ is the radius of the motor-driven pulley. Unlike traditional manipulators, CDMs utilize cables to drive their links and place motors at their base. The links are connected by hinges and cables. Leave out cables, and only moment exists between links of CDMs. Thus, ${ }^{i} f_{i}$ represents the force from link $i-1$ to link $i$, but ${ }^{\mathrm{i}} n_{\mathrm{i}}$ represents the moment of link $i$ exerted by its driving cable (numbered by $i$ ). The extra force caused by cable $j$ to link $i$ is denoted by ${ }_{j}^{i} F_{\mathrm{Cable}, i}$, and the pre-superscript indicates that the moment is measured in coordinate system $O_{i}$. The total number of DOF is $n$.

$$
{ }^{i} f_{i}={ }_{i+1}^{i} R{ }^{i+1} f_{i+1}+\sum_{j=i+1}^{n}{ }_{j}^{i} F_{\text {Cable }, i}+{ }^{i} F_{i} .
$$

When the cable passes through link guide pulleys, not only force ${ }^{i} F_{\text {Cable, } i}$ is induced, but also extra moment arises. The extra moment caused by cable $j$ to link $i$ is denoted by ${ }_{j}^{i} N_{\text {Cable }, i}$, and the pre-superscript indicates 
that the moment is measured in coordinate system $O_{i}$. The driving cable of link $i$ is numbered as cable $i$, and assume the manipulator has $n$ degrees of freedom. Calculate the moments relative to link $i$ 's mass center, sum them up, make the summation equal to zero, and one can obtain ${ }^{\mathrm{i}} n_{\mathrm{i}}$. One can get

$$
{ }^{i} N_{i}={ }^{i} n_{i}-{ }^{i} P_{C, i} \times{ }^{i} f_{i}-\left({ }^{i} P_{i+1}-{ }^{i} P_{C, i}\right) \times{ }^{i} f_{i+1},
$$

where ${ }^{i} N_{i}$ represents the moment of disturbance. Consider ${ }^{i} f_{i+1}={ }_{i+1}^{i} R^{i+1} f_{i+1}$ and substitute $i$ in eq. (13) by $i+1$. Then we get

$$
{ }^{i} n_{i}={ }^{i} N_{i}+\sum_{j=i+1}^{n}{ }_{j}^{i} N_{\text {Cable }, i}+{ }^{i} P_{C, i} \times{ }^{i} F_{i}+{ }^{i} P_{i+1} \times{ }_{i+1}^{i} R{ }^{i+1} f_{i+1} .
$$

The driving torque of the motor is given by

$$
\tau_{\mathrm{m}, i}=\frac{r_{\mathrm{m}}}{r_{\mathrm{j}}} \hat{n}_{i}
$$

where $\hat{n}_{i}$ denotes a certain element of $n_{i}$ corresponding to the axis of the joint. Usually, it is the third element on the condition that D-H method is utilized to establish the coordinate system.

In Figure 5, a segment of cable is taken for detailed analysis. A length of cable passes through a joint driving pulley to drive it through friction. Consequently, the tension of the cable varies along its length that contacts the pulley. Supposing the tension changes from $\tau_{1}$ to $\tau_{2}$, then the relationship between $\tau_{1}, \tau_{2}$ and $n_{i}$ is as follows

$$
n_{i}=r_{\mathrm{j}}\left(\tau_{2}-\tau_{1}\right)
$$

where $n_{i}$ is the torque provided by the cable to drive the corresponding link of the robot. Then a segment is taken for further analysis. Let us denote the force from pulley to cable by $\tau_{\mathrm{p}}$ and $\theta$ as the angle that rotates from $x$ axis to the centre of this segment. $\theta$ is positive if the rotation
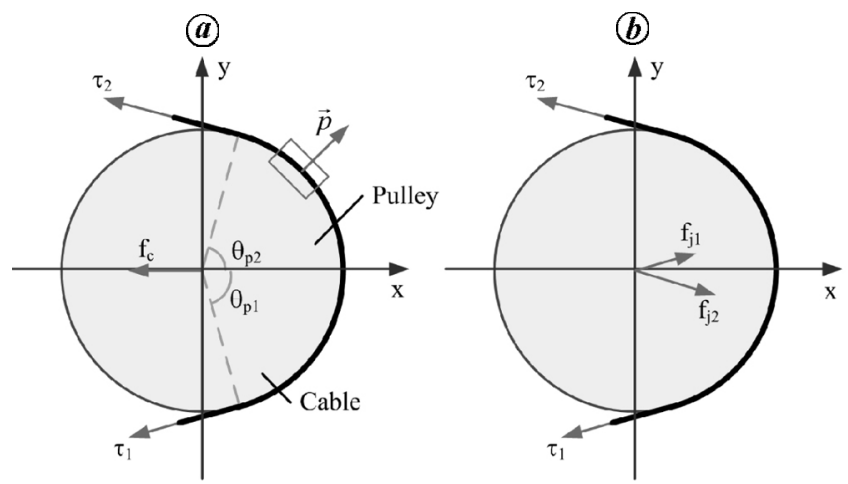

Figure 5. A segment of cable taken for analysis. is anti-clockwise, and it is negative if the rotation direction reverses. $\vec{p}$ is the force vector from the pulley to an arbitrary point on this contact segment of the cable. $\theta_{\mathrm{p} 1}$ and $\theta_{\mathrm{p} 2}$ are the corresponding central angles of the input and output points. These angles are measured between the radius of the pulley and $x$ axis, and have the same definition for positive and negative as $\theta$. Neglecting the mass of cable and considering force equilibrium of the contact cable, the resultant force from cable to the pulley can be expressed as

$$
f_{\mathrm{c}}=-\int_{\theta_{\mathrm{p} 1}}^{\theta_{\mathrm{p} 2}} p \mathrm{~d} \theta=\tau_{1}+\tau_{2}
$$

The relationship between $\theta_{\mathrm{p}}, \theta_{\mathrm{g} 0}$ and $\theta_{\mathrm{j} 0}$ is given by

$$
\theta_{\mathrm{p}}=\frac{\pi}{2}+\theta_{\mathrm{g} 0}=\pi-\theta_{\mathrm{j} 0}
$$

Then consider the forces in Figure 4; the cable is divided into three segments marked $1-3$. Cables 1 and 2 have constant tension; denote their tension by $\tau_{1}$ and $\tau_{2}$ respectively. Cable 3 has various tensions along its length. Forces between cables and links are shown by red arrows, and moment of joint-drive pulley is shown by an arc with arrowhead. These forces can be expressed in coordinate system $O_{i} x_{i} y_{i} z_{i}$ as follows

$$
\begin{aligned}
{\left[f_{i, 1}, f_{i, 2}, f_{i, 3}, f_{i, 4}\right] } & =\left[\begin{array}{ccc}
1-\cos \theta_{\mathrm{g} 0} & 0 & 0 \\
0 & \sin \theta_{\mathrm{g} 0} & 0 \\
0 & 0 & 1
\end{array}\right] \\
& \times\left[\begin{array}{cccc}
\tau_{1} & \tau_{2} & -\tau_{1} & -\tau_{2} \\
-\tau_{1} & \tau_{2} & -\tau_{1} & \tau_{2} \\
0 & 0 & 0 & 0
\end{array}\right] .
\end{aligned}
$$

Figure 4 is only a schematic diagram which cannot show the real direction of forces. One should refer to the results of calculation to get the direction of forces. $f_{i, 5}$ is given by

$$
f_{i, 5}=\left[\begin{array}{c}
\cos \theta_{\mathrm{g} 0}(\cos \theta+1)\left(\tau_{1}+\tau_{2}\right)+\sin \theta \sin \theta_{\mathrm{g} 0}\left(\tau_{2}-\tau_{1}\right) \\
\sin \theta_{\mathrm{g} 0}(\cos \theta-1)\left(\tau_{2}-\tau_{1}\right)-\sin \theta \cos \theta_{\mathrm{g} 0}\left(\tau_{1}+\tau_{2}\right) \\
0
\end{array}\right]
$$

Equation (15) can be used to calculate $n_{i+1}$ as driving torque for link $i+1$, but actually the interaction between cable and joint drive pulley is contact force and friction. The cable cannot directly provide driving torque and consequently, the hinges between links provide a force in the opposite direction of cable tensile force to form moment of couple force (Figure $5 \mathrm{~b}$ ). This serves as the driving torque for links instead of cable tensile force. The force 
provided by hinges is denoted by $f_{j 1}$ and $f_{j 2}$ corresponding to $\tau_{1}$ and $\tau_{2}$ respectively. Apparently, $\left|f_{j 1}\right|=\left|\tau_{1}\right|$ and $\left|f_{j 2}\right|=\left|\tau_{2}\right|$ holds. $f_{j 1}$ and $f_{j 2}$ are forces from link $i$ to link $i+1$, so counter-acting forces are applied on link $i$. These counter-acting forces are equal to $\tau_{1}$ and $\tau_{2}$ with different points of action. $f_{j 1}$ and $f_{j 2}$ are given by

$$
\begin{aligned}
& f_{j 1}=\left[\begin{array}{lll}
\tau_{1} \cos \theta_{\mathrm{g} 0} & -\tau_{1} \sin \theta_{\mathrm{g} 0} & 0
\end{array}\right]^{T}, \\
& f_{j 2}=\left[\begin{array}{lll}
\tau_{2} \cos \theta_{\mathrm{g} 0} & \tau_{2} \sin \theta_{\mathrm{g} 0} & 0
\end{array}\right]^{T} .
\end{aligned}
$$

${ }_{j}^{i} F_{\text {Cable }, i}$ is expressed as

$$
{ }_{j}^{i} F_{\text {Cable }, i}=\left\{\begin{array}{l}
\sum_{k=1}^{5}{ }^{i} f_{i, k}+{ }^{i} f_{j 1}+{ }^{i} f_{j 2}, \quad j=i+1, \\
\sum_{k=1}^{5}{ }^{i} f_{i, k}, \quad j>i+1 .
\end{array}\right.
$$

${ }_{i+1}^{i} N_{\text {Cable }, i}$ is given by

$$
\begin{aligned}
{ }^{i+1}{ }^{i} N_{\mathrm{Cable}, i} & ={ }^{i} \overrightarrow{O_{i} A} \times{ }_{j}^{i} f_{i, 1}+{ }^{i} \overline{O_{i} B} \times{ }_{j}^{i} f_{i, 2} \\
& +{ }^{i} \overline{O_{i} C} \times{ }_{j}^{i} f_{i, 3}+{ }^{i} \overline{O_{i} D} \times{ }_{j}^{i} f_{i, 4}-{ }^{i} P_{i+1} \times\left({ }^{i} f_{j 1}+{ }^{i} f_{j 2}\right) .
\end{aligned}
$$

Let us denote the length of link $i$ by $l_{i}$; then we get

$$
\begin{aligned}
& { }^{i} \overrightarrow{O_{i} A}=\left[d_{\mathrm{j} 0},-d_{\mathrm{g} 0}, 0\right]^{T}, \\
& { }^{i} \overline{O_{i} B}=\left[d_{\mathrm{j} 0}, d_{\mathrm{g} 0}, 0\right]^{T}, \\
& { }^{i} \overline{O_{i} C}=\left[l_{i}-d_{\mathrm{j} 0},-d_{\mathrm{g} 0}, 0\right]^{T}, \\
& { }^{i} \overline{O_{i} D}=\left[l_{i}-d_{\mathrm{j} 0}, d_{\mathrm{g} 0}, 0\right]^{T}, \\
& { }^{i} \overline{O_{i} V}=\left[l_{i}-r_{\mathrm{j}} \cdot \sin \theta_{\mathrm{g} 0}, r_{\mathrm{j}} \cdot \cos \theta_{\mathrm{g} 0}, 0\right]^{T}, \\
& { }^{i} \overline{O_{i} U}=\left[l_{i}-r_{j} \cdot \sin \theta_{\mathrm{g} 0},-r_{\mathrm{j}} \cdot \cos \theta_{\mathrm{g} 0}, 0\right]^{T} .
\end{aligned}
$$

Substitute eqs (26)-(31) into eq. (25) and simplify to get

$$
{ }_{i+1}^{i} N_{\text {Cable }, i}=\left[\begin{array}{lll}
0 & 0 & 0
\end{array}\right]^{T} .
$$

On the other hand

$$
{ }_{j}^{i} N_{\text {Cable }, i}={ }^{i} P_{i+1} \times\left({ }^{i} f_{j 1}+{ }^{i} f_{j 2}\right), \quad j>i+1 .
$$

So eq. (15) can be expressed as
${ }^{i} n_{i}={ }^{i} N_{i}+\sum_{j=i+2}^{n}{ }_{j}^{i} N_{\text {Cable }, i}+{ }^{i} P_{C, i} \times{ }^{i} F_{i}+{ }^{i} P_{i+1} \times{ }_{i+1}^{i} R{ }^{i+1} f_{i+1}$.

Finally, the impact of pretension is analysed. Compared with traditional manipulators, CDMs transfer power from the base to the joints by cables. It is obvious that CDMs cannot work without certain pretension, so it is necessary to know how pretension influences the dynamics of CDMs. Assume the manipulator has pretension $\tau_{0}$ in its cables and no other force exists. In other words, assume that the manipulator is isolated from other objects and is free from gravity. For the sake of simplicity and without loss of generality, only cable $n$ is considered for calculation, which is the driving cable of the distal joint of the manipulator. It can be calculated from eq. (34) that

$$
\begin{gathered}
{ }^{n} n_{n}={ }^{n} N_{n}+{ }^{n} P_{C, n} \times{ }^{n} F_{n}=\left[\begin{array}{lll}
0 & 0 & 0
\end{array}\right]^{T}, \\
{ }^{n-1} n_{n-1}={ }^{n-1} N_{n-1}+{ }^{n-1} P_{C, n-1} \times{ }^{n-1} F_{n-1} \\
+{ }^{n-1} P_{n} \times{ }_{n}^{n-1} R^{n} f_{n}=\left[\begin{array}{lll}
0 & 0 & 0
\end{array}\right]^{T} .
\end{gathered}
$$

Further, one can finally get

$$
{ }^{k} n_{k}=\left[\begin{array}{lll}
0 & 0 & 0
\end{array}\right]^{T}, \quad k=1,2, \ldots, n .
$$

Actually, no matter how much pretension is exerted on CDMs, it cannot drive the manipulator. In other words, pretension cannot perform the role of a driving force; so the result of eq. (37) is easy to understand. Consequently, when deducing dynamic formula of CDMs, pretension can be subtracted from cable stress for simplification. Here, an assumption that $\tau_{1}=0$ in eq. (17) is made for simplification. Under this assumption, $\tau_{2}$ is positive if it is larger than $\tau_{1}$, and vice versa. Then $\tau_{2}$ for joint $i$ is expressed as

$$
\tau_{2, i}=\frac{n_{i}}{r_{j}}
$$

Equation (20) can be written as

$\left[{ }^{i} f_{i, 1},{ }^{i} f_{i, 2},{ }^{i} f_{i, 3},{ }^{i} f_{i, 4}\right]=\frac{n_{i}}{r_{j}}\left[\begin{array}{cccc}0 & 1-\cos \theta_{\mathrm{g} 0} & 0 & \cos \theta_{\mathrm{g} 0}-1 \\ 0 & \sin \theta_{\mathrm{g} 0} & 0 & \sin \theta_{\mathrm{g} 0} \\ 0 & 0 & 0 & 0\end{array}\right]$. 
Then, eq. (24) is given by

$$
{ }_{j}^{i} F_{\mathrm{Cable}, i}=\left\{\begin{array}{l}
\frac{n_{i}}{r_{j}}\left[\begin{array}{c}
2 \cos \theta_{\mathrm{g} 0}+\cos \left(\theta-\theta_{\mathrm{g} 0}\right) \\
2 \sin \theta_{\mathrm{g} 0}-\sin \left(\theta-\theta_{\mathrm{g} 0}\right) \\
0
\end{array}\right], \quad j=i+1, \\
\frac{n_{i}}{r_{j}}\left[\begin{array}{c}
\cos \theta_{\mathrm{g} 0}+\cos \left(\theta-\theta_{\mathrm{g} 0}\right) \\
\sin \theta_{\mathrm{g} 0}-\sin \left(\theta-\theta_{\mathrm{g} 0}\right) \\
0
\end{array}\right], \quad j>i+1
\end{array}\right.
$$

Finally, eq. (33) can be expressed as

$$
{ }_{j}^{i} N_{\text {Cable }, i}=\frac{n_{i}}{r_{j}} \cdot{ }^{i} P_{i+1} \times\left[\begin{array}{lll}
\cos \theta_{\mathrm{g} 0} & \sin \theta_{\mathrm{g} 0} & 0
\end{array}\right]^{T}, \quad j>i+1 .
$$

\section{Parameter identification}

The recursive method has been used to calculate dynamics of CDMs. Based on this method, one can first calculate ${ }^{n} n_{n}$ and obtain the tension of cable $n$. Then ${ }^{n-1} n_{n-1}$ can be calculated, since the tension of cable $n$ is already known. Next, ${ }^{n-2} n_{n-2}$ can be calculated as the tension of both cable $n$ and cable $n-1$ is known. Step by step, all of the driving torque can be figured out and the complete dynamics model of CDM can be rearranged as follows

$$
\tau_{m, i}=M \ddot{\theta}_{i}+C\left(\theta_{i}, \dot{\theta}_{i}\right) \dot{\theta}_{i}+G\left(\theta_{i}\right) .
$$

Equation (42) can be further rewritten in the following form

$$
\begin{aligned}
& \tau_{m, i}=\left[\Phi_{1}\left(\theta_{i}, \dot{\theta}_{i}, \ddot{\theta}_{i}\right) \quad \Phi_{2}\left(\theta_{i}, \dot{\theta}_{i}, \ddot{\theta}_{i}\right) \quad \cdots \quad \Phi_{n}\left(\theta_{i}, \dot{\theta}_{i}, \ddot{\theta}_{i}\right)\right] \\
& \underbrace{}_{\substack{\text { Torque } \\
\text { matrix }}} \underbrace{}_{\text {Coefficient matrix }}
\end{aligned}
$$

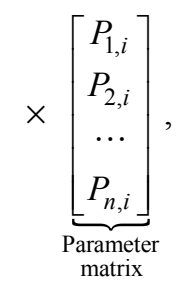

where $P_{1, i}, P_{2, i}, \ldots, P_{n, i}$ is the set of linearly independent parameters to be identified for link $i$. It is worth mentioning here that different links may have the same parameters. In other words, $P_{n, i}=P_{m, j}$ for link $i$ and link $j$. Considering unknown disturbance, however, the results of parameter identification may differ from theoretical calculations. During parameter identification, the parameter matrix is assumed to be unknown. In other words, the aim of this procedure is to derive the dynamic model of the manipulator without knowing its set-up. Therefore, although the results of parameter identification differ from those of theoretical calculation, one can accept the identification result and use it to derive a dynamic model of the manipulator if the identification process is reliable. During experiment or simulation, a series of $\tau_{m}$ and $\theta$ can be gathered pertime interval. Suppose a few groups of data are taken for identification; let us denote the total number of groups by $p$. Each group of data is gathered per time interval and contains a set of values consisting of $\tau_{m}, \theta, \dot{\theta}, \ddot{\theta}$. Then the parameters to be identified can be calculated by

$$
\begin{aligned}
{\left[\begin{array}{c}
P_{1, i} \\
P_{2, i} \\
\cdots \\
P_{n, i}
\end{array}\right] } & {\left[\begin{array}{cccc}
\Phi_{11}\left(\theta_{i}, \dot{\theta}_{i}, \ddot{\theta}_{i}\right) & \Phi_{21}\left(\theta_{i}, \dot{\theta}_{i}, \ddot{\theta}_{i}\right) & \cdots & \Phi_{n 1}\left(\theta_{i}, \dot{\theta}_{i}, \ddot{\theta}_{i}\right) \\
\Phi_{12}\left(\theta_{i}, \dot{\theta}_{i}, \ddot{\theta}_{i}\right) & \Phi_{22}\left(\theta_{i}, \dot{\theta}_{i}, \ddot{\theta}_{i}\right) & \cdots & \Phi_{n 2}\left(\theta_{i}, \dot{\theta}_{i}, \ddot{\theta}_{i}\right) \\
\ldots & \ldots & \cdots & \cdots \\
\Phi_{1 p}\left(\theta_{i}, \dot{\theta}_{i}, \ddot{\theta}_{i}\right) & \Phi_{2 p}\left(\theta_{i}, \dot{\theta}_{i}, \ddot{\theta}_{i}\right) & \cdots & \Phi_{n p}\left(\theta_{i}, \dot{\theta}_{i}, \ddot{\theta}_{i}\right)
\end{array}\right] } \\
& \times\left[\begin{array}{c}
\tau_{m, i, 1} \\
\tau_{m, i, 2} \\
\ldots \\
\tau_{m, i, p}
\end{array}\right],
\end{aligned}
$$

where $\Phi_{r s}\left(\theta_{i}, \dot{\theta}_{i}, \ddot{\theta}_{i}\right)$ denotes $\Phi_{r}\left(\theta_{i}, \dot{\theta}_{i}, \ddot{\theta}_{i}\right)$ in data group numbered by $s ; \tau_{m, i, s}$ denotes $\tau_{m, i}$ in data group numbered by $s .[\bullet]^{+}$denotes Moore-Penrose pseudo-inverse of $\bullet$. The coefficient matrix contains $\ddot{\theta}$, which is easily influenced by disturbance; so a well-designed identification trajectory for the experiment is essential. An identification trajectory containing several terms in Fourier series is utilized

$$
\hat{\theta}_{d}=\sum_{k=1}^{c} \hat{a}_{k} \cos (k \hat{w} t)+\sum_{k=1}^{c} \hat{b}_{k} \sin (k \hat{w} t)
$$

where $\hat{w}$ is a positive number and $c$ is a positive integer. These terms are restricted by the performance of the manipulator. Moreover, the coefficient matrix should have small condition number which measures the sensitivity of the solution of linear equations to errors in the data. Here the condition number of a matrix is equal to the largest singular value of the matrix divided by its smallest value. Joints of manipulators have limitation in their rotation or translation ranges. Furthermore, speed and acceleration of the driving motors are limited. All of these restrictions pose limitation to the identification trajectory; so eq. (45) and its derivation are restricted by the following conditions.

$$
\theta_{d, \text { min }} \leq \hat{\theta}_{d} \leq \theta_{d, \max },\left|\frac{\mathrm{d}}{\mathrm{d} t} \hat{\theta}_{d}\right| \leq \omega_{d, \max },\left|\frac{\mathrm{r}^{2}}{\mathrm{~d} t^{2}} \hat{\theta}_{d}\right| \leq \alpha_{d, \text { max }}
$$

where $\theta_{d, \min }$ and $\theta_{d, \max }$ are extreme positions of rotation or translation of the joint. $\omega_{d, \max }$ and $\alpha_{d, \max }$ are maximum 


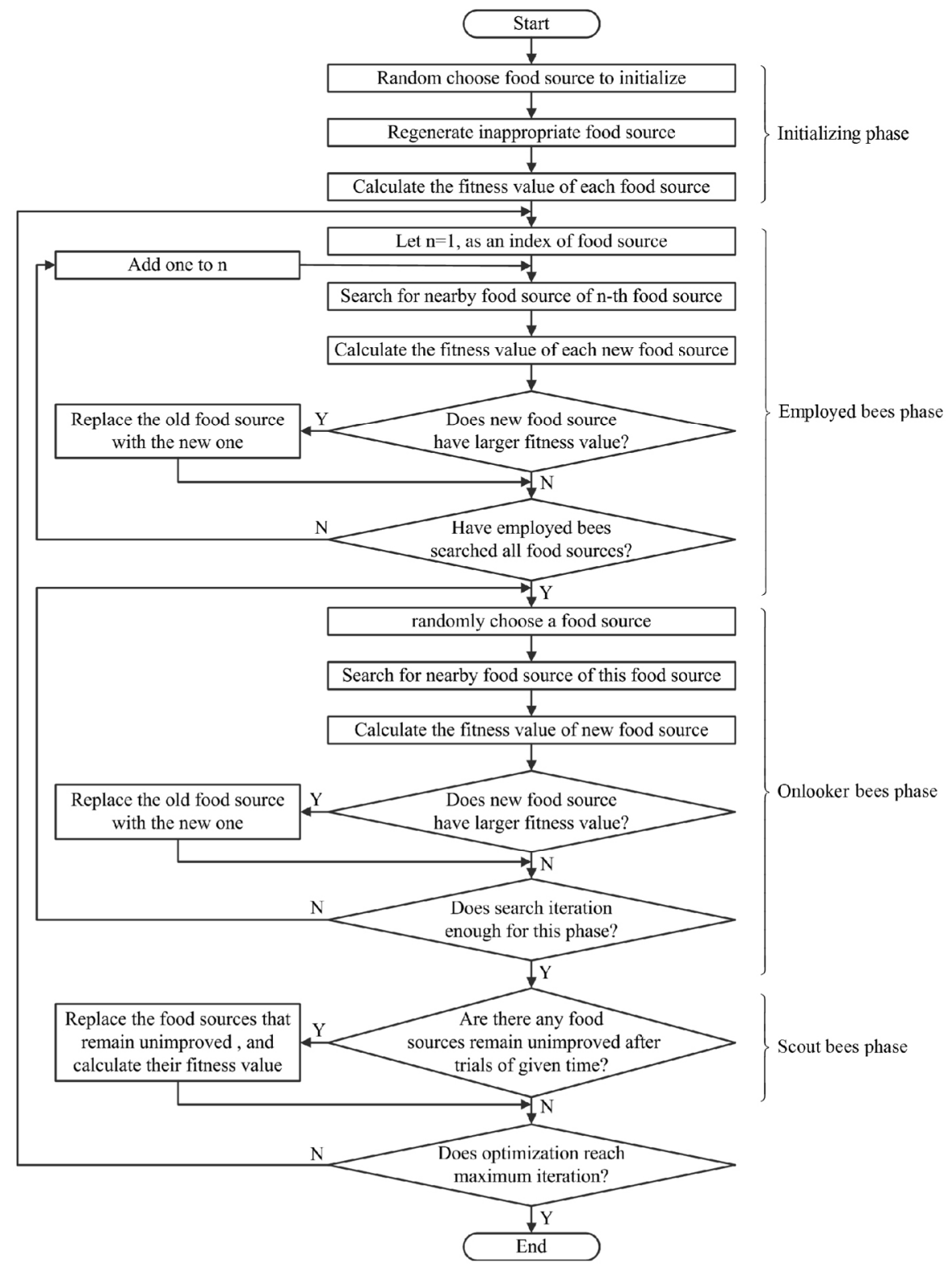

Figure 6. Flow chart of artificial bee colony algorithm.

joint speed and maximum joint acceleration respectively. It is inevitable that some errors exist between desired trajectory and actual trajectory; so while determining $\theta_{d, \text { min }}, \theta_{d, \text { max }}, \omega_{d, \text { max }}$ and $\alpha_{d, \text { max }}$, a margin of safety should be considered. In order to make $\hat{\theta}_{d}=0$ at the starting time, take $n=c-1$ and eq. (45) can be rewritten as

$$
\begin{aligned}
\hat{\theta}_{d} & =\sum_{k=1}^{n} \hat{a}_{k} \cos (k \hat{w} t)+\sum_{k=1}^{n} \hat{b}_{k} \sin (k \hat{w} t) \\
& -\sum_{k=1}^{n} \hat{a}_{k} \cdot \cos (n \hat{w} t+\hat{w} t)-\sum_{k=1}^{n} \hat{b}_{k} \cdot \sin (n \hat{w} t+\hat{w} t) .
\end{aligned}
$$

Moreover, eq. (47) should not take too large numbers as its coefficients; therefore

$$
\begin{aligned}
& |w| \leq w_{\max },\left|\hat{a}_{k}\right| \leq a_{\max },\left|\hat{b}_{k}\right| \leq b_{\max }, k=1,2, \ldots, n, \\
& \left|\sum_{k=1}^{n} \hat{a}_{k}\right| \leq a_{\max },\left|\sum_{k=1}^{n} \hat{b}_{k}\right| \leq b_{\max }, k=1,2, \ldots, n .
\end{aligned}
$$

Based on the aforementioned restriction, artificial bee colony algorithm is utilized to calculate the coefficients in eq. (47) to ensure that the coefficient matrix in eq. (44) has a small condition number as possible. Detailed procedure of artificial bee colony algorithm can be found in Yan et $a l .{ }^{52}$. In the present study, 'food source' is a set of 
$\hat{a}_{k}, \hat{b}_{k}, \quad k=1,2, \ldots, n$. When generating candidates of food source, eq. (48) acts as both the upper limit and lower limit. If the generated candidate does not satisfy eq. (49), the algorithm will be discarded and a new one generated. The objective function is the sum of two parts; one part is the condition number of coefficient matrix in eq. (43). A number much larger than the condition number of coefficient matrix will be added to the objective function if the desired trajectory does not satisfy eq. (46). Thus, the other part of the objective function is zero on condition that eq. (46) is satisfied, or a large number on condition that eq. (46) is violated. The fitness function can be obtained by adding 1 to the objective function and calculating the reciprocal of the sum. Figure 6 shows the complete procedure to get $\hat{a}_{k}, \hat{b}_{k}, k=1,2, \ldots, n$ utilizing artificial bee colony algorithm.

\section{Numerical example and simulation}

First, the dynamics formula of a 2-DOF CDM is derived utilizing the aforementioned method and follows

$$
\begin{aligned}
& n_{1}(3)=\ddot{\theta}_{1}\left({ }^{C} I_{1}(3,3)+\frac{1}{4} l_{1}^{2} m_{1}+l_{1}^{2} m_{2}\right) \\
& +\frac{1}{2} l_{1} l_{2} m_{2} c \theta_{2}\left(\ddot{\theta}_{1}+\ddot{\theta}_{2}\right)-\frac{1}{2} l_{1} l_{2} m_{2} s \theta_{2}\left(\dot{\theta}_{1}^{2}+\dot{\theta}_{2}^{2}\right) \\
& -\dot{\theta}_{1} \dot{\theta}_{2} l_{1} l_{2} m_{2} s \theta_{2}+g l_{1} c \theta_{1}\left(\frac{1}{2} m_{1}+m_{2}\right), \\
& n_{2}(3)=\left(\ddot{\theta}_{1}+\ddot{\theta}_{2}\right)\left({ }^{C} I_{2}(3,3)+\frac{1}{4} l_{2}^{2} m_{2}\right) \\
& +\frac{1}{2} \ddot{\theta}_{1} l_{1} l_{2} m_{2} c \theta_{2}+\frac{1}{2} \dot{\theta}_{1}^{2} l_{1} l_{2} m_{2} s \theta_{2}+\frac{1}{2} g l_{2} m_{2} c \theta_{12},
\end{aligned}
$$

where $\quad s \theta_{1}=\sin \left(\theta_{1}\right), \quad c \theta_{1}=\cos \left(\theta_{1}\right), \quad s \theta_{2}=\sin \left(\theta_{2}\right)$, $c \theta_{2}=\cos \left(\theta_{2}\right)$ and $c \theta_{12}=\cos \left(\theta_{12}\right) . \quad n_{i}(3)$ is the third element of $n_{i}$ which corresponds to the driving torque of link $i$. Similarly, ${ }^{C} I_{1}(3,3)$ denotes the element at the third row and third column of ${ }^{C} I_{1}$. Equations (6) and (16) describe the torque and rotation angle of driving motors.

Actual trajectory may differ from $\theta_{d}$ given in eq. (45); so one needs to use the actual trajectory to modify $\hat{a}_{k}$, $\hat{b}_{k}$ and $\hat{w}$ in eq. (45). This procedure is similar to curvefitting and the real trajectory can be expressed as

$$
\theta=\sum_{k=1}^{n} a_{k} \cos (k w t)+\sum_{k=1}^{n} b_{k} \sin (k w t),
$$

where $w$ is the actual moving frequency; $a_{k}$ and $b_{k}$ are actual coefficients for terms of the Fourier series. $\dot{\theta}_{i}$ and $\ddot{\theta}_{i}$ can be derived from eq. (52).
A 2-DOF CDM has been designed and simulated using ADAMS and MATLAB/Simulink. Table 1 shows geometry parameters of CDM. $\theta_{\mathrm{g} 0}$ and $\theta_{\mathrm{j} 0}$ are calculated using eqs (1) and (2), and are also shown in Table 1. The mass centre of this links and inertia reference point are both situated at the geometry centres.

By substituting the geometry parameters of CDM into the dynamic formula (50) and (51), we get

$$
\begin{aligned}
\tau_{m, 1} & =0.6 \ddot{\theta}_{1}+0.18 c \theta_{2}\left(\ddot{\theta}_{1}+\ddot{\theta}_{2}\right) \\
& -0.18 s \theta_{2}\left(\dot{\theta}_{1}^{2}+\dot{\theta}_{2}^{2}\right)-0.36 \dot{\theta}_{1} \dot{\theta}_{2} s \theta_{2}+11.76 c \theta_{1}, \\
\tau_{m, 2} & =0.12\left(\ddot{\theta}_{1}+\ddot{\theta}_{2}\right)+0.18 \ddot{\theta}_{1} c \theta_{2}+0.18 \dot{\theta}_{1}^{2} s \theta_{2} \\
& +2.94 \cos \left(\theta_{1}+\theta_{2}\right),
\end{aligned}
$$

where angles are in radians, and $\tau_{m, 1}$ and $\tau_{m, 2}$ are in $\mathrm{N} \cdot \mathrm{m}$.

Four cases are simulated and analysed to show the validity of the dynamic model given by eqs (53) and (54).

Case 1: Choose sine wave as the desired trajectory; only use PID controller.

Case 2: Choose the same desired trajectory as in case 1; use PID controller with dynamic model feed-forward.

Case 3: Choose the desired trajectory constituted by segments of quadratic polynomial; only use PID controller.

Case 4: Choose the same desired trajectory as in case 3; use PID controller with dynamic model feed-forward.

Figure 7 shows the detailed control schemes of these four cases. In the figure, ' $u$ PID' and ' $u$ _model' represent the control input of PID controller and dynamic model feedforward respectively. $u$ represents the control input that passes to the ADAMS software.

Table 1. Geometry parameters of a cable-driven manipulator (CDM)

\begin{tabular}{lc}
\hline Parameter & Value \\
\hline$l_{1}$ & $0.6 \mathrm{~m}$ \\
$l_{2}$ & $0.6 \mathrm{~m}$ \\
$m_{1}$ & $2 \mathrm{~kg}$ \\
$m_{2}$ & $1 \mathrm{~kg}$ \\
$d_{\mathrm{g} 0}$ & $0.022 \mathrm{~m}$ \\
$d_{\mathrm{j} 0}$ & $0.124 \mathrm{~m}$ \\
$r_{\mathrm{j}}$ & $0.1 \mathrm{~m}$ \\
$r_{\mathrm{m}}$ & $0.1 \mathrm{~m}$ \\
$r_{\mathrm{g}}$ & $0.02 \mathrm{~m}$ \\
$\theta_{\mathrm{g} 0}$ & $62.277^{\circ}$ \\
$\theta_{\mathrm{j} 0}$ & $27.723^{\circ}$ \\
${ }^{C} I_{1}$ & $\operatorname{diag}(0.01,0.06,0.06) \mathrm{kg} \mathrm{m}^{2}$ \\
${ }^{C} I_{2}$ & $\operatorname{diag}(0.003,0.03,0.03) \mathrm{kg} \mathrm{m}^{2}$ \\
\hline
\end{tabular}




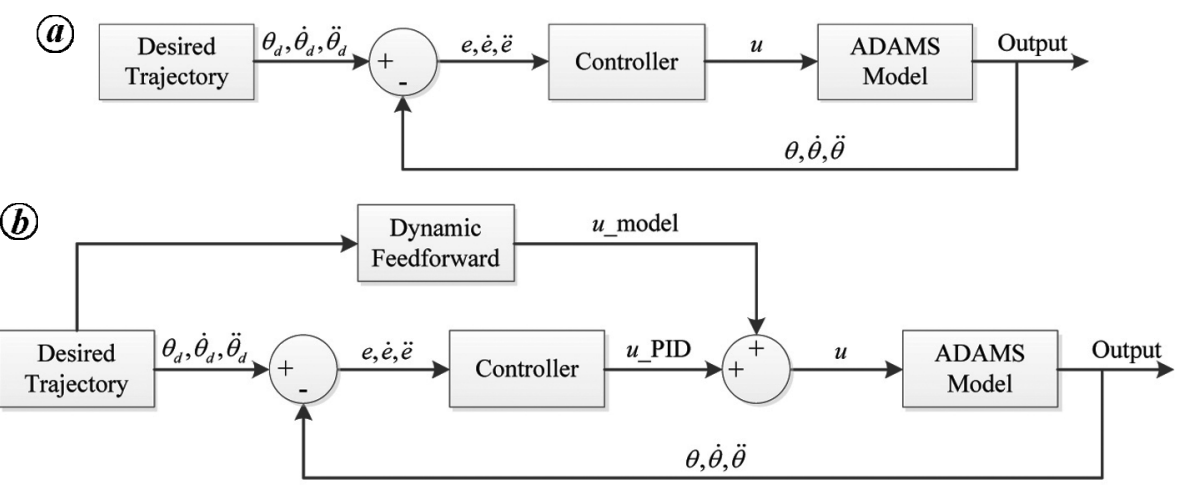

Figure 7. Detailed control scheme of four cases. $\boldsymbol{a}$, Cases 1 and 3 ; $\boldsymbol{b}$, Cases 2 and 4.

(a)

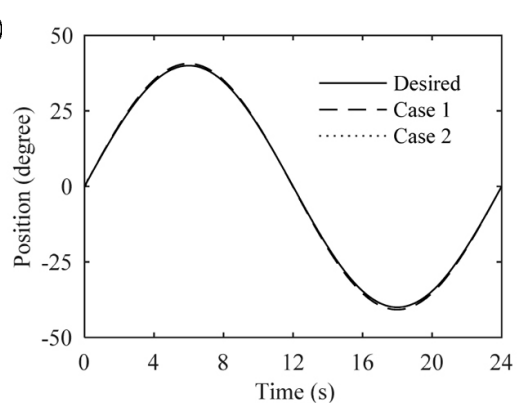

(c)
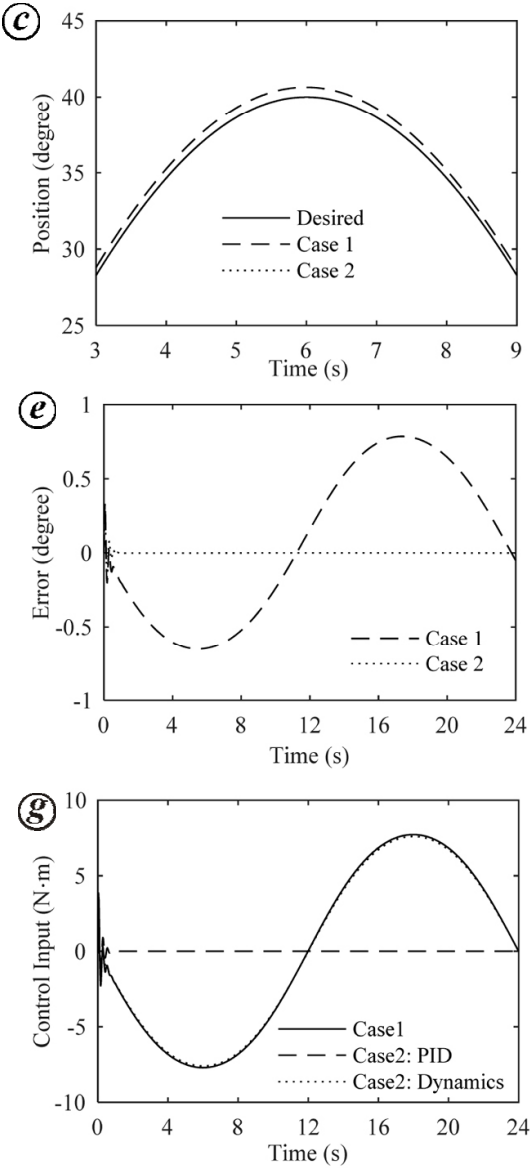

(b)
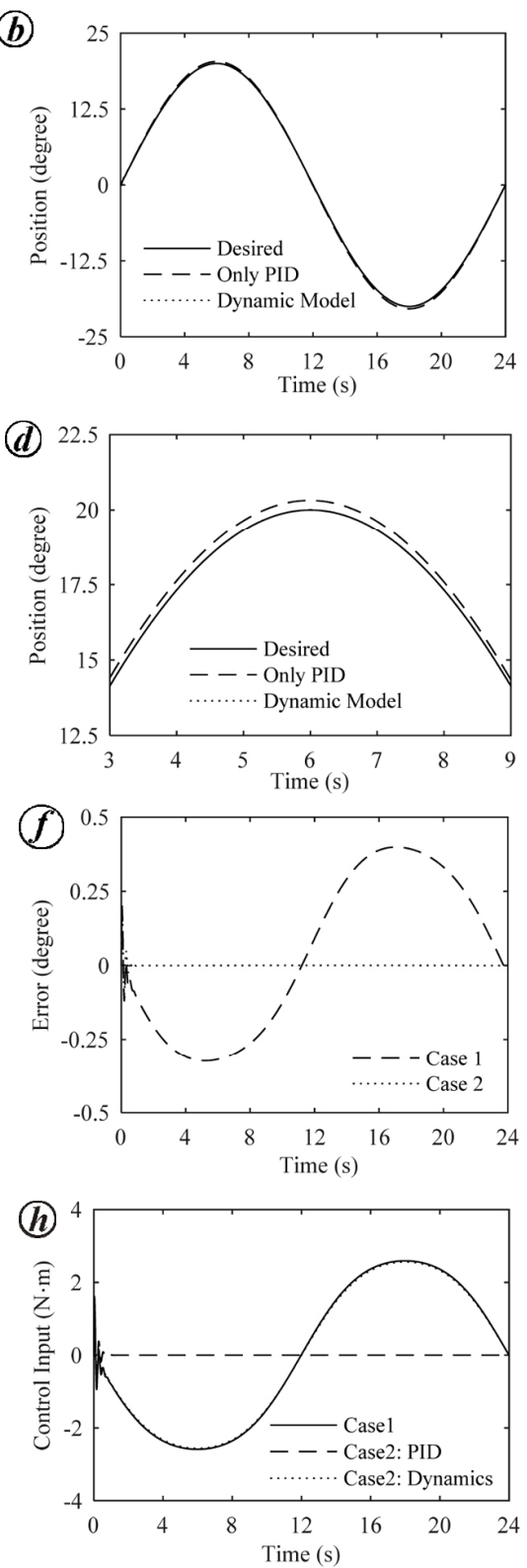

Figure 8. Control performance of cases 1 and 2. a, Position of joint 1. b, Position of joint 2. $\boldsymbol{c}$, Positioning of joint 1 ( $3-9$ s). $\boldsymbol{d}$, Positioning of joint $2(3-9 \mathrm{~s})$. $\boldsymbol{e}$, Tracking error of joint $1 . \boldsymbol{f}$, Tracking error of joint $2 . \boldsymbol{g}$, Control input of joint 1 . $\boldsymbol{h}$, Control input of joint 2 . 

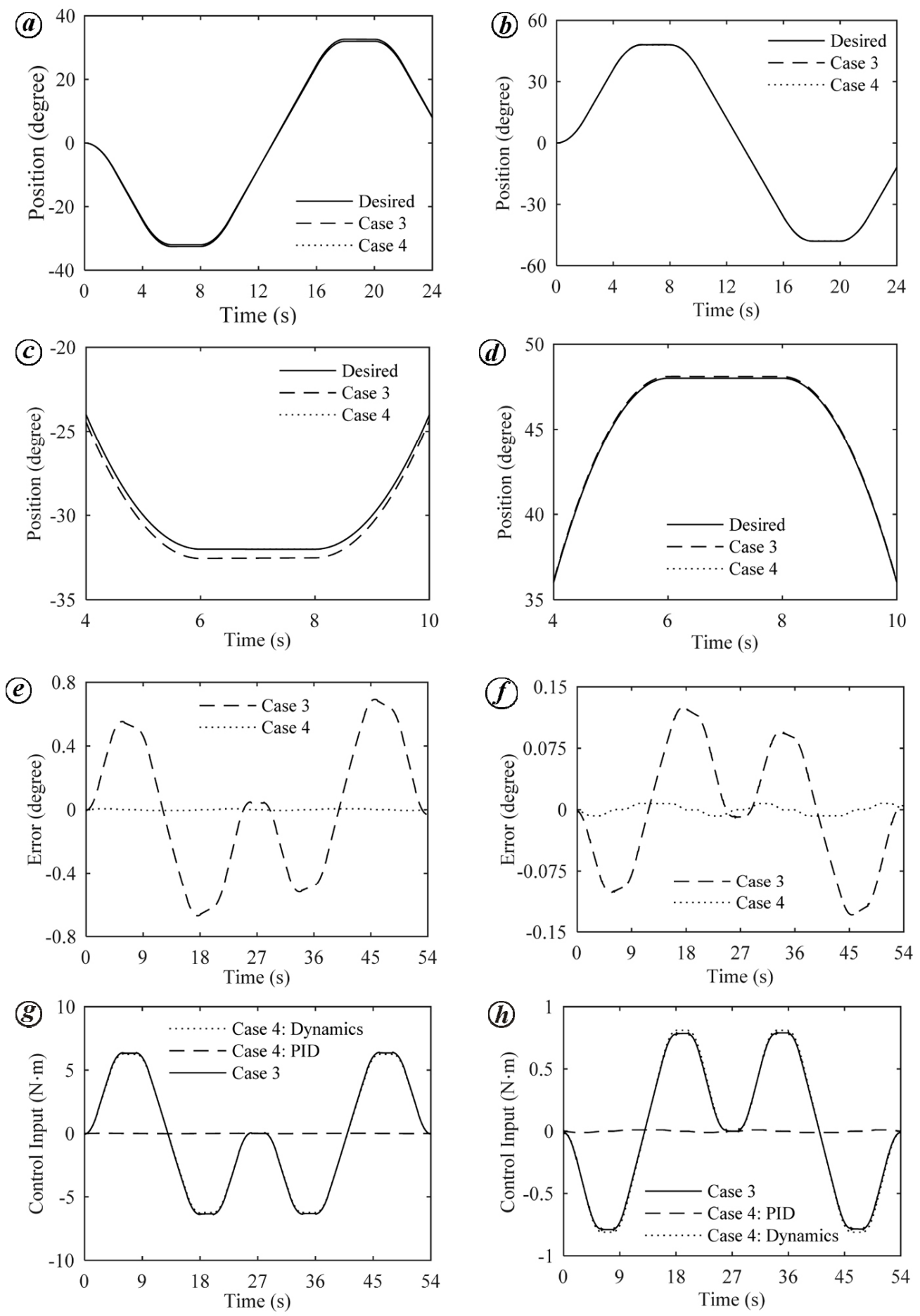

Figure 9. Control performance of cases 3 and 4. a, Position of joint 1. b, Position of joint 2. $\boldsymbol{c}$, Positioning of joint 1 (4-10 s). $\boldsymbol{d}$, Positioning of joint $2(4-10 \mathrm{~s}) . \boldsymbol{e}$, Tracking error of joint $1 . \boldsymbol{f}$, Tracking error of joint $2 . \boldsymbol{g}$, Control input of joint $1 . \boldsymbol{h}$, Control input of joint 2.

Figures 8 and 9 show the simulation results of these cases. For the sake of comparison, control input of cases 2 and 4 are separated by two parts: one part is the control input of PID controller and the other part is the control input of dynamic model feed-forward, marked by 'PID' and 'Dynamics' respectively. One can observe that in all of the four cases, joints of the manipulator can track the desired trajectory. However, cases 2 and 4 have much less tracking error compared with cases 1 and 3, which shows the advantage of dynamic feed-forward. Moreover, the control input of dynamic model feed-forward in cases 2 and 4 has minor differences compared with the control 


\section{RESEARCH ARTICLES}

input of PID controller. Besides, the control input of PID controller in cases 2 and 4 is almost zero. Thus, the proposed dynamic model can precisely calculate the required control input of CDM, which confirms the validity of the proposed dynamic model.

Next, a parameter identification experiment of 2-DOF CDM is carried out. The dynamic model obtained by parameter identification is compared with theoretical calculations.

For the identification procedure, eqs (50) and (51) are rewritten as follows

$$
\begin{aligned}
& \tau_{m, 1}= \\
& {\left[\ddot{\theta}_{1} \frac{1}{2} c \theta_{2}\left(\ddot{\theta}_{1}+\ddot{\theta}_{2}\right)-\frac{1}{2} s \theta_{2}\left(\dot{\theta}_{1}^{2}+\dot{\theta}_{2}^{2}\right)-\dot{\theta}_{1} \dot{\theta}_{2} s \theta_{2} \quad g \cdot c \theta_{1}\right]} \\
& \times\left[\begin{array}{c}
{ }^{C} I_{1}+0.25 l_{1}^{2} m_{1}+l_{1}^{2} m_{2} \\
l_{1} l_{2} m_{2} \\
l_{1}\left(0.5 m_{1}+m_{2}\right)
\end{array}\right], \\
& \tau_{m, 2}=\left[\begin{array}{lll}
\ddot{\theta}_{1}+\ddot{\theta}_{2} & \frac{1}{2} \ddot{\theta}_{1} c \theta_{2}+\frac{1}{2} \dot{\theta}_{1}^{2} s \theta_{2} \quad \frac{1}{2} g \cdot c \theta_{12}
\end{array}\right] \\
& \times\left[\begin{array}{c}
{ }^{C} I_{2}+0.25 l_{2}^{2} m_{2} \\
l_{1} l_{2} m_{2} \\
l_{2} m_{2}
\end{array}\right] .
\end{aligned}
$$

Denote

$$
\begin{aligned}
& {\left[\begin{array}{l}
P_{1,1} \\
P_{2,1} \\
P_{3,1}
\end{array}\right]=\left[\begin{array}{c}
{ }^{C} I_{1}+0.25 l_{1}^{2} m_{1}+l_{1}^{2} m_{2} \\
l_{1} l_{2} m_{2} \\
l_{1}\left(0.5 m_{1}+m_{2}\right)
\end{array}\right],} \\
& {\left[\begin{array}{l}
P_{1,2} \\
P_{2,2} \\
P_{3,2}
\end{array}\right]=\left[\begin{array}{c}
{ }^{C} I_{2}+0.25 l_{2}^{2} m_{2} \\
l_{1} l_{2} m_{2} \\
l_{2} m_{2}
\end{array}\right] .}
\end{aligned}
$$

The coefficient matrix in eq. (43) is given by

$$
\begin{aligned}
& {\left[\Phi_{1}\left(\theta_{1}, \dot{\theta}_{1}, \ddot{\theta}_{1}\right) \quad \Phi_{2}\left(\theta_{1}, \dot{\theta}_{1}, \ddot{\theta}_{1}\right) \quad \Phi_{3}\left(\theta_{1}, \dot{\theta}_{1}, \ddot{\theta}_{1}\right)\right]} \\
& =\left[\begin{array}{ll}
\ddot{\theta}_{1} & \frac{1}{2} c \theta_{2}\left(\ddot{\theta}_{1}+\ddot{\theta}_{2}\right)-\frac{1}{2} s \theta_{2}\left(\dot{\theta}_{1}^{2}+\dot{\theta}_{2}^{2}\right)-\dot{\theta}_{1} \dot{\theta}_{2} s \theta_{2} \quad g \cdot c \theta_{1}
\end{array}\right],
\end{aligned}
$$

$$
\begin{aligned}
& {\left[\Phi_{1}\left(\theta_{2}, \dot{\theta}_{2}, \ddot{\theta}_{2}\right) \quad \Phi_{2}\left(\theta_{2}, \dot{\theta}_{2}, \ddot{\theta}_{2}\right) \quad \Phi_{3}\left(\theta_{2}, \dot{\theta}_{2}, \ddot{\theta}_{2}\right)\right]}
\end{aligned}
$$

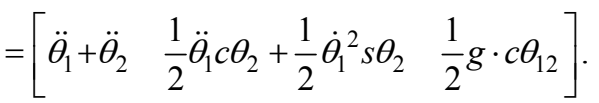

The identification trajectory is optimized by artificial bee colony algorithm; Table 2 shows the optimization set up. Figure 10 shows the objective function values during the optimization process. The optimized identification trajectory is given as follows and a $60 \mathrm{~s}$ segment of the same is shown in Figure 11.

$$
\begin{aligned}
\hat{\theta}_{d 1}= & 0.0174 \cos (0.345 t)+0.00872 \cos (0.69 t) \\
& -0.00194 \cos (1.04 t)-0.0242 \cos (1.38 t) \\
& -0.0114 \sin (0.345 t)+0.0234 \sin (0.69 t) \\
& -0.0122 \sin (1.04 t)+\left(2.0 \times 10^{-4}\right) \sin (1.38 t), \\
\hat{\theta}_{d 2}= & -0.113 \cos (0.345 t)+0.0717 \cos (0.69 t) \\
& +0.0146 \cos (1.04 t)+0.0267 \cos (1.38 t) \\
& -0.0233 \sin (0.345 t)-0.0197 \sin (0.69 t) \\
& -0.00164 \sin (1.04 t)+0.0446 \sin (1.38 t) .
\end{aligned}
$$

Then a PID controller is used to control the CDM to move along the identification trajectory. The total simulation time is $300 \mathrm{~s}$. Considering the periodicity of the identification trajectory, only $0-60 \mathrm{~s}$ of the control input in the identification experiment is shown in Figure 12.

The actual trajectory of the CDM is fitted into the form of eq. (52) and results are given as follows

$$
\begin{aligned}
\hat{\theta}_{d 1}= & 0.0177 \cos (0.345 t)+0.00893 \cos (0.69 t) \\
& -0.00198 \cos (1.04 t)-0.0247 \cos (1.38 t) \\
& -0.0117 \sin (0.345 t)+0.0239 \sin (0.69 t) \\
& -0.0125 \sin (1.04 t)+\left(2.35 \times 10^{-4}\right) \sin (1.38 t), \\
\hat{\theta}_{d 2}= & -0.114 \cos (0.345 t)+0.0723 \cos (0.69 t) \\
& +0.0147 \cos (1.04 t)+0.0267 \cos (1.38 t) \\
& -0.0235 \sin (0.345 t)-0.0197 \sin (0.69 t) \\
& -0.00176 \sin (1.04 t)+0.045 \sin (1.38 t) .
\end{aligned}
$$

Considering that there exists relatively large tracking error at the beginning of the simulation, the results before $10 \mathrm{~s}$ are discarded. A hundred data points are gathered per second during simulation to form the coefficient matrix in eq. (43). Corresponding data points of control 
input are also recorded to form the torque matrix in eq. (43). Finally, the dynamic parameter of CDM is calculated using eq. (44) as follows

$$
\begin{aligned}
& {\left[\begin{array}{lll}
P_{1,1} & P_{2,1} & P_{3,1}
\end{array}\right]=\left[\begin{array}{lll}
0.5997 & 0.3501 & 1.2007
\end{array}\right]^{T},} \\
& {\left[\begin{array}{lll}
P_{1,2} & P_{2,2} & P_{3,2}
\end{array}\right]=\left[\begin{array}{lll}
0.1195 & 0.3686 & 0.6001
\end{array}\right]^{T} .}
\end{aligned}
$$

Parameters calculated mathematically using eqs (57) and (58) are given by

$$
\begin{aligned}
& {\left[\begin{array}{lll}
\hat{P}_{1,1} & \hat{P}_{2,1} & \hat{P}_{3,1}
\end{array}\right]=\left[\begin{array}{lll}
0.6000 & 0.3600 & 1.2000
\end{array}\right]^{T},} \\
& {\left[\begin{array}{lll}
\hat{P}_{1,2} & \hat{P}_{2,2} & \hat{P}_{3,2}
\end{array}\right]=\left[\begin{array}{lll}
0.1200 & 0.3600 & 0.6000
\end{array}\right]^{T} .}
\end{aligned}
$$

Except for the middle term of the parameter matrix, the identification results are almost equal to the mathematically calculated ones. From eqs (59) and (60), one may find that the middle term of the coefficient matrix is rather complex. Consequently, it is difficult to obtain precise value of the middle term of the coefficient matrix,

Table 2. Optimization set-up

\begin{tabular}{lc}
\hline Parameter & Value \\
\hline$\theta_{d, \min }$ & $-\mathrm{pi} / 6$ \\
$\theta_{d, \max }$ & $\mathrm{pi} / 6\left(^{\circ}\right)$ \\
$\omega_{d, \max }$ & $\mathrm{pi} / 24(\% / \mathrm{s})$ \\
$\alpha_{d, \max }$ & $\mathrm{pi} / 48\left(\% / \mathrm{s}^{2}\right)$ \\
$w_{\max }$ & $\mathrm{pi} / 6(\mathrm{rad} / \mathrm{s})$ \\
$a_{\max }$ & 1 \\
$b_{\max }$ & 1 \\
Number of colony size & 20 \\
Number of food sources & 10 \\
Maximum number of cycles & 2000 \\
Number of optimized parameters & 13 \\
\hline
\end{tabular}

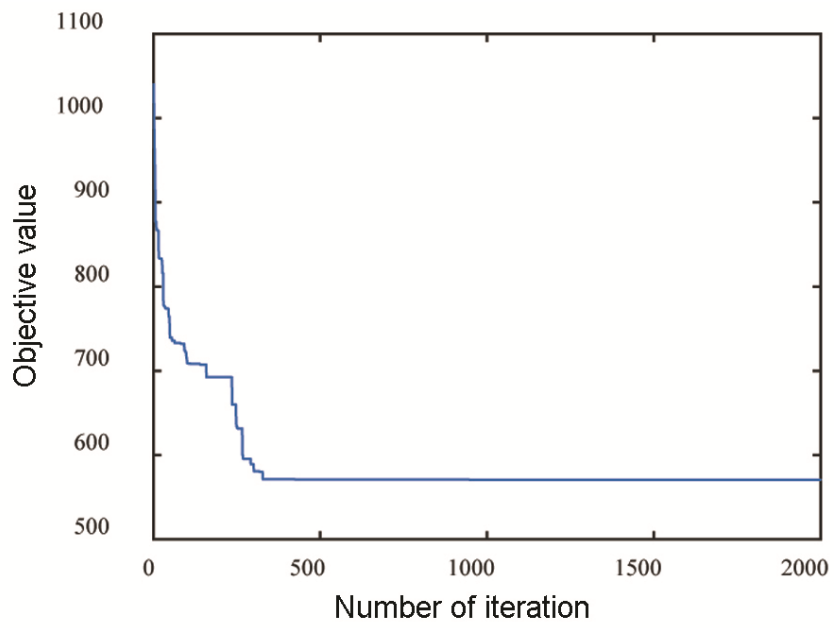

Figure 10. Objective function value during optimizing process. which will introduce relatively large error to the corresponding term of the parameter matrix. Therefore, generally speaking, the identification results are close to the mathematically calculated ones; the dynamic model and parameter identification method are valid.

\section{Conclusion}

The present study describes the basic structure of CDM and deduces some of their geometric relationships. Then Newton-Euler recursive method is discussed as a basic method to obtain dynamic model of CDM. Pulleys of $\mathrm{CDM}$ are categorized by joint guide pulley, link guide pulley and joint drive pulley. Interaction forces between cables and these kinds of pulleys are examined. For easy application of the recursive method, the impact of pretension in cables to dynamics is analysed. Next, a parameter identification method for dynamic model of CDM is proposed. A trajectory consisting of several terms of the Fourier series is used as the identification trajectory. In

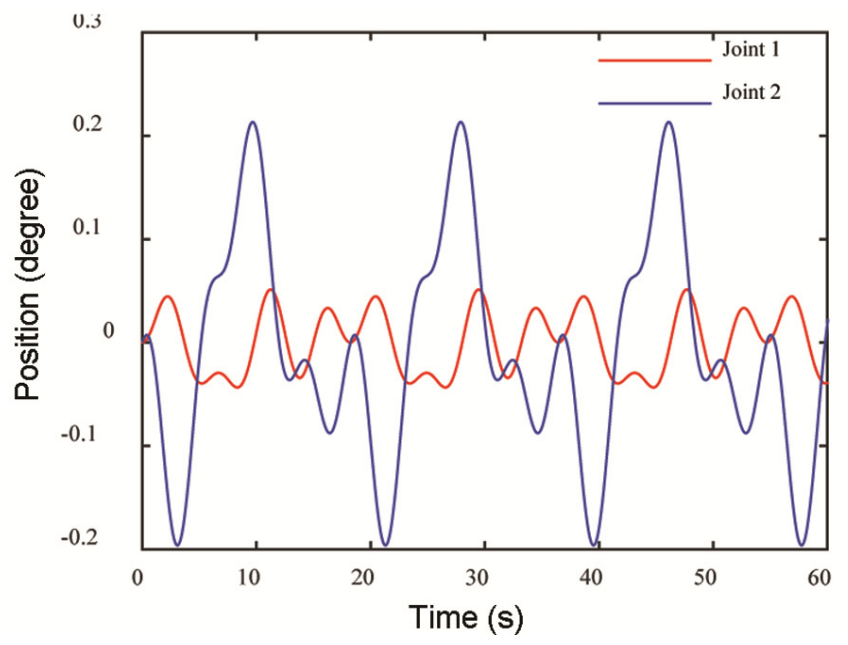

Figure 11. Optimized identification trajectory.

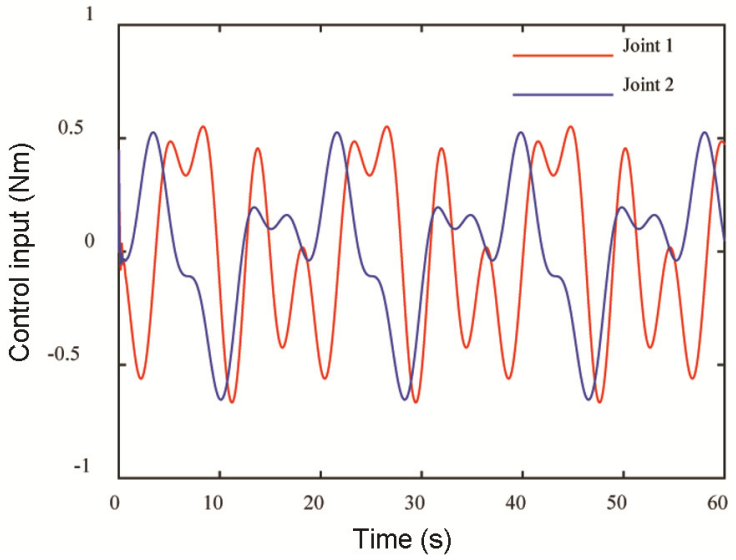

Figure 12. Control input in the identification experiment. 


\section{RESEARCH ARTICLES}

parameter identification, the coefficient matrix should have a small condition number as possible. However, restrictions to the identification trajectory pose challenges to obtain the optimal trajectory. Artificial bee colony algorithm is applied to get the best identification trajectory under various restrictions. Finally, simulations verify the correctness of the proposed dynamic model and validity of the parameter identification method.

1. Shang, W. et al., Synchronization control in the cable space for cable-driven parallel robots. IEEE Trans. Ind. Electron., 2018, 66(6), 4544-4554; doi:10.1109/TIE.2018.2864512.

2. Zhang, N., Shang, W. W. and Cong, S., Geometry-based trajectory planning of a 3-3 cable-suspended parallel robot. IEEE Trans. Robot., 2017, 33(2), 484-491.

3. Wang, Y. et al., Practical tracking control of robot manipulators with continuous fractional-order nonsingular terminal sliding mode. IEEE Trans. Ind. Electron., 2016, 63(10), 6194-6204.

4. Wang, Y. et al., A new adaptive time-delay control scheme for cable-driven manipulators. IEEE Trans. Ind. Inform., doi:10.1109/ TII.2018.2876605.

5. Wang, Y. et al., Practical adaptive fractional-order nonsingular terminal sliding mode control for a cable-driven manipulator. Int. J. Robust Nonlinear Control, 2019, 29(5), 1396-1417; doi: $10.1002 /$ rnc.4441.

6. Wang, Y. et al., Adaptive super-twisting fractional-order nonsingular terminal sliding mode control of cable-driven manipulators. ISA Trans., 2019, 86, 163-180; doi:10.1016/j.isatra.2018.11.009.

7. Wang, Y. et al., Trajectory tracking control of underwater vehiclemanipulator system using discrete time delay estimation. IEEE Access, 2017, 5, 7435-7443; doi:10.1109/ACCESS.2017.2701350.

8. Du, J. et al., Jacobian analysis of a long-span cable-driven manipulator and its application to forward solution. Mech. Mach. Theory, 2010, 45(9), 1227-1238.

9. Duan, X. et al., Calibration and motion control of a cable-driven parallel manipulator based triple-level spatial positioner. $A d v$. Mech. Eng., 2014, 6, 1-10.

10. Tzemanaki, A. et al., Design of a multi-DOF cable-driven mechanism of a miniature serial manipulator for robot-assisted minimally invasive surgery. In IEEE International Conference Biomedical Robotics and Biomechatronics, Singapore, 2016, pp. $55-60$.

11. Gonzalez-Rodriguez, A. et al., On the effects of the design of cable-driven robots on kinematics and dynamics models accuracy. Mechatronics, 2017, 43, 18-27.

12. Xue, R. et al., A cable-pulley system modeling based position compensation control for a laparoscope surgical robot. Mech. Mach. Theory, 2017, 118, 283-299.

13. Gungor, G. et al., Online estimation and compensation of friction in industrial cable robot manipulation. IFAC-PapersOnLine, 2015, 48(3), 1332-1337.

14. Grossard, M. and Fichera, F., Polynomial piece-wise stiffness in cable-based transmissions for robots: modeling and identification. IFAC-PapersOnLine, 2017, 50(1), 14581-14587.

15. Fichera, F. and Grossard, M., On the modeling and identification of stiffness in cable-based mechanical transmissions for robot manipulators. Mech. Mach. Theory, 2017, 108, 176-190.

16. Yu, L. et al., Dynamical model and experimental identification of a cable-driven finger joint for surgical robot. In IEEE International Conference Mechanical Automation, Takamatsu, Japan, 2017; doi:10.1109/ICMA.2017.8015860.

17. Qi, Z., Wang, J. and Wang, G., An efficient model for dynamic analysis and simulation of cable-pulley systems with time-varying cable lengths. Mech. Mach. Theory, 2017, 116, 383-403.
18. Zhang, N. and Shang, W., Dynamic trajectory planning of a 3DOF under-constrained cable-driven parallel robot. Mech. Mach. Theory, 2016, 98, 21-35.

19. Ismail, M., Lahouar, S. and Romdhane, L., Collision-free and dynamically feasible trajectory of a hybrid cable-serial robot with two passive links. Robot. Auton. Syst., 2016, 80, 24-33.

20. Yuan, M. et al., An improved on-line trajectory planner with stability-guaranteed critical test curve algorithm for generalized parametric constraints. IEEE/ASME Trans. Mechatron., 2018, 23(5), 2459-2469.

21. Zhou, J. P., Zhong, Z. D. and Wang, Z. Z., Motion trajectory planning of 3-dof wire-driven parallel robot. Adv. Mater. Res., 2012, 424, 369-372.

22. Ismail, M., Samir, L. and Romdhane, L., Dynamic in path planning of a cable driven robot. Design Model. Mech. Syst., 2013.

23. Pinto, A. M. et al., A cable-driven robot for architectural constructions: a visual-guided approach for motion control and pathplanning. Auton. Robots, 2017, 41(7), 1-13.

24. Lahouar, S. et al., Collision free path-planning for cable-driven parallel robots. Robotics Autonom, Syst., 2009, 57(11), 1083-1093.

25. Tang, L. et al., Dynamic trajectory planning study of planar twodof redundantly actuated cable-suspended parallel robots. Mechatronics, 2015, 30, 187-197.

26. Lau, D., Oetomo, D. and Halgamuge, S. K., Inverse dynamics of multilink cable-driven manipulators with consideration of joint interaction forces and moments. IEEE Trans. Robot., 2015, 31(2), $479-488$.

27. Xuechao, D., Yuanying, Q. and Jingli, D., Dynamic analysis and vibration attenuation of cable-driven parallel manipulators for large workspace applications. Adv. Mech. Eng., 2013, 4, 1-6.

28. Nielsen, M. C. et al., Constrained multi-body dynamics for modular underwater robots - theory and experiments. Ocean Eng., 2018, 149, 358-372.

29. Meng, W. et al., Consensus control of nonlinear multiagent systems with time-varying state constraints. IEEE Trans. Cybern., 2017, 47(8), 2110-2120.

30. Meng, W. et al., Distributed control of high-order nonlinear input constrained multiagent systems using a backstepping-free method. IEEE Trans. Cybern., 2018, 99, 1-11; doi:10.1109/TCYB.2018. 2853623.

31. Yuan, M. et al., Time optimal contouring control of industrial biaxial gantry: a highly efficient analytical solution of trajectory planning. IEEE/ASME Trans. Mechatron., 2017, 22(1), 247-257.

32. Deng, W., Yao, J. and Ma, D., Robust adaptive precision motion control of hydraulic actuators with valve dead-zone compensation. ISA Trans., 2017, 70, 269-278.

33. Deng, W., Yao, J. and Ma, D., Time-varying input delay compensation for nonlinear systems with additive disturbance: an output feedback approach. Int. J. Robust Nonlinear Control, 2018, 28(1), $31-52$.

34. Chen, Z., Yao, B. and Wang, Q., Accurate motion control of linear motors with adaptive robust compensation of nonlinear electromagnetic field effect. IEEE/ASME Trans. Mechatron., 2013, 18(3), 1122-1129.

35. Chen, Z., Yao, B. and Wang, Q., $\mu$-Synthesis based adaptive robust control of linear motor driven stages with high-frequency dynamics: a case study with comparative experiments. IEEE/ASME Trans. Mechatron., 2015, 20(3), 1482-1490.

36. Chen, Z., Pan, Y. J. and Gu, J., Integrated adaptive robust control for multilateral teleoperation systems under arbitrary time delays. Int. J. Robust Nonlinear Control, 2016, 26(12), 2708-2728.

37. Chen, J. et al., Research on fuzzy control of path tracking for underwater vehicle based on genetic algorithm optimization. Ocean Eng., 2018, 156, 217-223.

38. Kong, K. and Tomizuka, M., Proxy-based impedance control of a cable-driven assistive system for upper extremity rehabilitation. IFAC Proc. Vol., 2011, 44(1), 2871-2876. 
39. Kong, K., Proxy-based impedance control of a cable-driven assistive system. Mechatronics, 2013, 23(1), 147-153.

40. Babaghasabha, R., Khosravi, M. A. and Taghirad, H. D., Adaptive robust control of fully-constrained cable driven parallel robots. Mechatronics, 2014, 25, 27-36.

41. Caverly, R. J. and Forbes, J. R., State estimator design for a single degree of freedom cable-actuated system. J. Franklin Inst., 2016, 353(18), 4845-4869.

42. Jung, Y. and Bae, J., An asymmetric cable-driven mechanism for force control of exoskeleton systems. Mechatronics, 2016, 40, 41-50.

43. Boehler, Q. et al., From modeling to control of a variable stiffness device based on a cable-driven tensegrity mechanism. Mech. Mach. Theory, 2017, 107, 1-12.

44. Wang, Y. et al., A new continuous fractional-order nonsingular terminal sliding mode control for cable-driven manipulators. $A d v$. Eng. Software, 2018, 119, 21-29.

45. Wang, Y. et al., Time delay control of cable-driven manipulators with adaptive fractional-order nonsingular terminal sliding mode. Adv. Eng. Software, 2018, 121, 13-25.

46. Wang, Y. et al., A new discrete time delay control of hydraulic manipulators. Proc. iMeche, Part I, 2017, 231(3), 168-177.

47. Wang, Y., Chen, B. and Wu, H., Joint space tracking control of underwater vehicle-manipulator systems using continuous nonsingular fast terminal sliding mode. Proc. Inst. Mech. Eng. Part M. J. Eng. Maritime Environ., 2018, 232(4), 448-458; doi:10.1177/ 1475090217742241.
48. Wang, Y., Chen, B. and Wu, H., Practical continuous fractionalorder nonsingular terminal sliding mode control of underwater hydraulic manipulators with valve deadband compensators. Proc. Inst. Mech. Eng. Part M, 2018; doi:10.1177/1475090217753900.

49. Jin, M., Lee, J. and Tsagarakis, N. G., Model-free robust adaptive control of humanoid robots with flexible joints. IEEE Trans. Indust. Electron., 2017, 64(2), 1706-1715.

50. Hartenberg, R. S. and Denavit, J., A kinematic notation for lower pair mechanisms based on matrices. J. Appl. Mech., 1955, 77(2), $215-221$.

51. Xu, W. et al., Kinematic analysis of a newly designed cable-driven manipulator. Trans. Can. Soc. Mech. Eng., 2018, 42(2), 125-135.

52. Yan, F. et al., Time delay control of cable-driven manipulators with artificial bee colony algorithm. Trans. Can. Soc. Mech. Eng., 2018, 42(2), 177-186.

ACKNOWLEDGEMENTS. This work was supported by the National Natural Science Foundation of China (51705243, 51575256), Natural Science Foundation of Jiangsu Province (BK20170789), and China Postdoctoral Science Foundation (2018M630552).

Received 16 November 2018; revised accepted 9 January 2019

doi: $10.18520 / \mathrm{cs} / \mathrm{v} 116 / \mathrm{i} 8 / 1331-1345$ 